\title{
On the Role of Scales in Elastomer Friction
}

\author{
Valentin L. Popov ${ }^{1,2,3, a)}$, Sergey Psakhie ${ }^{2,3,4, b)}$, and Mikhail Popov ${ }^{1,2, c)}$ \\ ${ }^{1}$ Berlin University of Technology, Berlin, 10623, Germany \\ ${ }^{2}$ National Research Tomsk State University, Tomsk, 634050, Russia \\ ${ }^{3}$ National Research Tomsk Polytechnic University, Tomsk, 634050, Russia \\ ${ }^{4}$ Institute of Strength Physics and Materials Science SB RAS, Tomsk, 634055, Russia

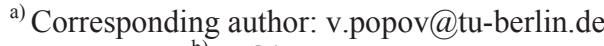 \\ b) sp@ispms.tsc.ru \\ c)mpopov@fastmail.fm
}

\begin{abstract}
The paper is devoted to a general analysis of friction of elastomers from the point of view of scales contributing to the force of friction. We argue that - contrary to the wide spread opinion - elastomer friction is not a multiscale phenomenon, but is governed mostly by the interplay of only two scales - the largest and the smallest scales of roughness of the contacting bodies. This is illustrated by analyzing the main ideas of the theory of elastomer friction based on the paradigm of Greenwood, Tabor and Grosch. The same conclusions can be obtained from the widely used contact theory proposed by Persson.
\end{abstract}

Keywords: elastomers, friction, fractal surfaces, macroscale, microscale

\section{INTRODUCTION}

In practical analysis of engineered systems, the simplest friction law of Amontons [1] is usually used, stating that the force of friction, $F$, is proportional to the normal force, $F_{\mathrm{n}}: F=\mu F_{\mathrm{n}}$, the proportionality coefficient $\mu$ being called coefficient of friction. It is no secret that Amontons" "law" is only a very rough zeroth-order approximation of real friction. Already Coulomb knew that the coefficient of sliding friction depends on sliding velocity and normal force and that static friction depends explicitly on time [2]. However, even 230 years after Coulomb no generalized laws of friction exist that could be reliably used in engineering practice. It is often argued that the reason for this lies in the multi-scale nature of friction, and that all scales necessarily have to be included into consideration to achieve a realistic model of friction [3]. In the present paper we will argue that this is not the case: In friction and in other phenomena associated with contact of rough surfaces - as in most other physical processes - there exist characteristic scales which provide the main contribution to the quantities of interest. Therefore, as in most areas of physics, the most productive approach is searching for the relevant scales and studying them. The present work is devoted to an analysis of the contribution to friction of different scales for one class of materials — elastomers with linear rheology.

\section{BASICS OF ELASTOMER FRICTION}

Since Greenwood and Tabor [4], and especially after the classic work of Grosch [5], it has been widely accepted that elastomer friction is mostly due to internal dissipative losses in material that are caused by deformation through surface asperities of the counter body. The force of friction typically increases with velocity, reaches a plateau and decreases again (Fig. 1). The plateau is normally of most practical interest. Physically, the behavior of an elastomer in this range is dominated by the lost modulus of the elastomer [6], and the elastomer behaves roughly speaking as a simple fluid. In particular, the relaxation of the elastomer after indentation or ploughing by an asperity is very slow.

International Conference on Physical Mesomechanics of Multilevel Systems 2014

AIP Conf. Proc. 1623, 507-510 (2014); doi: 10.1063/1.4898993

(C) 2014 AIP Publishing LLC 978-0-7354-1260-6/\$30.00 


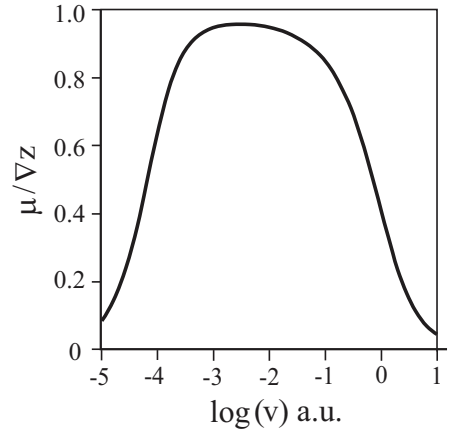

FIGURE 1. Typical dependency of the coefficient of friction on sliding velocity

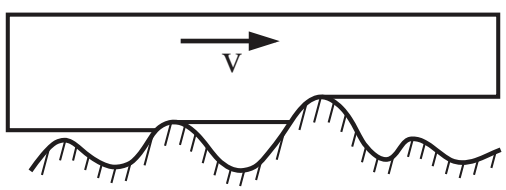

FIGURE 2. Configuration of the contact of a viscoelastic material with a rough surface in the region of the plateau of the coefficient of friction

Practically all microcontacts are therefore one-sided as shown in Fig. 2. It is easy to understand that the coefficient of friction is then equal to the one-sided average of the slope of the surface profile.

Despite the apparent simplicity of this physical picture, already at this point an interesting and non-trivial question arises: What is the average slope of the profile? Let us start the discussion of this question with an estimation of the rms value of the gradient of the surface profile over the whole surface. The average gradient of the surface profile, $\nabla z$, can be expressed as an integral of the spectral power density of the surface profile, $C(q),[7]$ according to

$$
\left\langle\nabla z^{2}\right\rangle \approx \int C(q) q^{3} \mathrm{~d} q
$$

It is known that many natural surfaces have the property of fractality, and for randomly self-affine, fractal surfaces the spectral power density is known to be a power-function of the wave-vector $q$ :

$$
C_{2 \mathrm{D}}(q)=\text { const } \cdot\left(q / q_{0}\right)^{-2 H-2}
$$

where $H$ is the Hurst-exponent and $q_{0}$ some reference wave-vector. For typical Hurst exponents smaller than one, the resulting integral diverges at the upper limit of integration. This means, that for a true fractal surface (without an upper cut-off wave vector), the surface gradient is infinitely large. In practice, of course, there is always some upper cut-off wave vector $q_{\max }$, and the surface gradient is determined by one or two orders of magnitude of wave vectors

at and below $q_{\max }$. In other words, for typical surfaces that we are dealing with, the friction force is determined by the roughness components with the largest wave-vectors (or the smallest scale of the system). One can say that understanding friction is equivalent to understanding the nature of this smallest relevant scale.

Of course, it is the surface slope in the contact region and not over the whole surface which is determining the coefficient of friction at the plateau. As has been shown already by Archard in 1950th [8], that the main effect of changing normal force is the number of asperities coming into contact, while the local conditions in the real contact area, including the surface gradient, depend only weakly on the normal force. However, this relatively weak dependence is exactly what we would now like to discuss in more detail. The actual surface gradient is a function of the current contact configuration. In the next section we will argue that the governing parameter for the contact configuration is the indentation depth $d$.

\section{INDENTATION DEPTH AS A GOVERNING PARAMETER OF CONTACT CONFIGURATION}

If a rigid body of an arbitrary shape is pressed against a homogeneous elastic half-space then the resulting contact configuration is only a function of the indentation depth $d$. At a given indentation depth, the contact configuration does not depend on the elastic properties of the medium, and will be the same even for indentation of a viscous fluid or of any linearly viscoelastic material. This general behavior was recognized by Lee and Radok $[9,10]$ and was verified numerically for fractal rough surfaces [11]. Further, the contact configuration at a given depth 
remains approximately invariant for media with thin coatings [12] and for multi-layered systems, provided the difference of elastic properties of the different layers is not too large [13]. In [14], it was argued that this is equally valid for media which are heterogeneous in the lateral direction (along the contact plane). Along with the contact configuration, all contact properties including the real contact area, the contact length, the contact stiffness, as well as the rms value of the surface gradient in the contact area will be unambiguous functions of the indentation depth. The indentation depth is thus a convenient und robust "governing parameter" for contact and frictional properties of media with linear rheology. Note, that this is equally valid for tangential contact. This can easily be illustrated with the example of contact of a rigid body with an incompressible elastic half-space: For a circular contact with an arbitrary radius $a$, the ratio of the normal stiffness $k_{z}$ and the tangential stiffness $k_{x}$ is constant and given by the Cattaneo-Mindlin factor $[15,16]$ for incompressible media $k_{z} / k_{x}=1.5$. From this follows that for a frictional contact with the coefficient of friction $\mu$, the maximum tangential displacement to the onset of complete sliding is determined solely by the indentation depth and is equal to $u_{x, \max }=1.5 \mu d[17,18]$. This result does not depend on the form of the body and is valid for arbitrary bodies of revolution and even for randomly rough fractal surfaces [19].

In practice, however, the controlled and measured quantity is normally not the indentation depth but the normal force. The latter is connected with the indentation depth through the contact stiffness. The contact stiffness is, however, known to be determined almost entirely by the long wave-length part of the spectral density of the surface profile (i.e. the macroscopic form of the contacting bodies) and does not depend on the detailed topography on the microscale [20]. We therefore come to the important conclusion, that frictional force is determined mainly by the smallest spatial scale while its weak dependence on the normal force is governed almost exclusively by the largest spatial scale of the system. This two-scale picture of frictional processes opens new effective ways for the physical analysis of friction.

\section{TWO-SCALE NATURE OF CONTACT BETWEEN FRACTAL SURFACES}

The two-scale nature of contacts with self-affine fractal surfaces can also be illustrated from another point of view. Many contact problems can be approximately described by the very popular contact theory by B. N. J. Persson et al. [21]. According to this theory, any contact property - contact area, contact stiffness, thermal or electrical conductivity, adhesive force or quality of seals and so on can be described in terms of integrals of the spectral power density $C(q)$ multiplied with different powers of the wave vector $q$. All these integrals have the form $\int q^{-2 H-1} q^{\alpha} \mathrm{d} q$ and diverge either on the upper or on the lower integration limit. This means that the main contribution to the integrals is given either by the region near the lower cut-off wave vector or the region near the upper cut-off. The form of the power spectrum in the intermediate range of wave vectors does not play any essential role. The only exceptions are cases where the integrated function is $q^{-2 H-1} q^{\alpha} \propto q^{-1}$ in which case the integral logarithmically diverges both on the upper and on the lower limit. Thus, according to Persson's theory, any problems with contacts of self-affine fractal surfaces have to be either two-scale problems or have a weak, logarithmic dependency on both the microscopic and macroscopic scales.

In conclusion, we propose that the multi-scale view of friction adds very little to the accuracy of friction models, while making them significantly more complicated. The most important processes in friction happen at two distinct scales. Therefore any model that correctly describes the contact stiffness on the largest scale and reproduces the gradient of the surface profile on the smallest scale, is eligible for the study of frictional processes. Examples of models based on the two-scale nature of frictional contacts can be found in $[22,23]$.

\section{ACKNOWLEDGEMENT}

This work was supported in part by the Ministry of Education of the Russian Federation, the Deutsche Forschungsgemeinschaft (DFG) and by COST Action MP1303.

\section{REFERENCES}

1. G. Amontons, "De la resistance cause'e dans les machines, tant par let frottements des parties qui les component, que par la roideur des cordes qu'on y employe, et la maniere de calculer l'un et l'autre", Memoire de l'Academie Royale 19 (1699). 
2. Ch. A. Coulomb, Theorie des Machines Simple, Nouvelle Edition (Bachelier, Paris, 1821).

3. B. N. J. Persson, J. Phys: Condens. Matter. 18, 7789 (2006).

4. J. A. Greenwood and D. Tabor, Proc. Roy. Soc. London 71, 989 (1958).

5. K. A. Grosch, Proc. Roy. Soc. London A. Math. Phys. Sci. 274, 21 (1963).

6. V. L. Popov, Contact Mechanics and Friction. Physical Principles and Applications (Springer-Verlag, 2010).

7. R. Pohrt, Normal Stiffness of Multiscale Rough Surfaces in Elastic Contact, PhD Thesis, TU Berlin, 2013.

8. J. F. Archard, Proc. R. Soc. A 243, 190 (1957).

9. E. H. Lee, Quart. Appl. Math. 13, 183 (1955).

10. J. R. M. Radok, Quart. Appl. Math. 15, 198 (1957).

11. S. Kürschner and V. L. Popov, Phys. Rev. E 87, 042802 (2013).

12. I. I. Argatov and F. J. Sabina, Int. J. Eng. Sci. 50, 132 (2012).

13. H. J. Gao, C. H. Chiu, and J. Lee, Int. J. Solids Struct. 29, 2471 (1992).

14. V. L. Popov, Phys. Mesomech. 16, 97 (2013).

15. C. Cattaneo, "Sul contatto di due corpi elastici: distribuzione locale degli sforzi", Rendiconti dell Accademia Nazionale dei Lincei., 27, 342-348, 434-436, 474-478 (1938).

16. R. D. Mindlin, ASME J. Appl. Mech., 16, 259 (1949).

17. V. L. Popov and M. Hess, Facta Universitatis. Mech. Eng. 12, 1 (2014).

18. V. L. Popov and M. Heß, Method of Dimensionality Reduction in Contact Mechanics and Friction (Springer 2014).

19. B. Grzemba, R. Pohrt, E. Teidelt, and V. L. Popov, Wear 309(1), 256 (2014).

20. C. Campana, B. N. J. Persson, and M. H. Müser, J. Phys. Condens. Matter 23, 085001 (2011).

21. B. N. J. Persson, O. Albohr, U. Tartaglino, A. I. Volokitin, and E. Tosatti, J. Phys. Condens. Matter 17, R1 (2005).

22. Q. Li, M. Popov, A. Dimaki, A. E. Filippov, S. Kürschner, and V. L. Popov, Phys. Rev. Lett., 111, 034301 (2013).

23. Q. Li, A. Dimaki, M. Popov, S. G. Psakhie, and V. L. Popov, Sci. Rep. 4, 5795 (2014). 